\title{
Modified McKeown procedure with uniportal thoracoscope for upper or middle esophageal cancer: initial experience and preliminary results
}

\author{
Qi Wang, Wei Ping, Yixin Cai, Shengling Fu, Xiangning Fu, Ni Zhang \\ Department of Thoracic Surgery, Tongji Hospital, Tongji Medical College, Huazhong University of Science and Technology, Wuhan 430030, China \\ Contributions: (I) Conception and design: Q Wang, Y Cai; (II) Administrative support: X Fu, S Fu; (III) Provision of study materials or patients: \\ X Fu, N Zhang; (IV) Collection and assembly of data: Q Wang, W Ping; (V) Data analysis and interpretation: Q Wang, W Ping, N Zhang; \\ (VI) Manuscript writing: All authors; (VII) Final approval of manuscript: All authors. \\ Correspondence to: Ni Zhang. Department of Thoracic Surgery, Tongji Hospital, Tongji Medical College, Huazhong University of Science and \\ Technology, 1095 Jie Fang Avenue, Wuhan 430030, China. Email: zhangnidoc@vip.163.com.
}

\begin{abstract}
Background: Uniportal video-assisted thoracic surgery (VATS) resections are of increasing interest in many thoracic surgery departments. With each experience in VATS lobectomy, we have incrementally improved the less invasive techniques in esophagectomy. Here, we report the preliminary results and feasibility of a modified McKeown procedure with uniportal thoracoscopy for upper or middle esophageal cancer in our institution.

Methods: Between March 2015 and May 2016, modified uniportal McKeown procedure with uniportal thoracoscopy for upper or middle esophageal cancer was attempted in 44 patients in our institution.

Results: Of the patients treated with uniportal thoracoscopy and laparoscopy, no patients were converted to open procedures, and all had a complete resection. The mean operative time was $408 \pm 34$ min (range, 394$495 \mathrm{~min}$ ). The mean thoracic operation was $163 \pm 16 \mathrm{~min}$ (range, 135-199 min). The mean blood loss was $245 \pm 102 \mathrm{~mL}$ (range, 100-450 mL). The mean number of lymph nodes resected was 24 (range, 14-36). The mean ventilator usage of the patients after surgery was $0.3 \pm 0.6$ days, and the mean intensive care stay was 1.6 days (range, 1 to 7 days). The mean hospital stay was 11.8 days (range, 7 to 22 days). Major complications developed in 2 patients, both of whom had to undergo tracheotomy due to respiratory failure. No patients died of complications postoperatively, and none had clinically significant anastomotic leaks.

Conclusions: Modified McKeown minimally invasive esophagectomy (MIE) with uniportal thoracoscopy seems to be a feasible option for patients with upper or middle esophageal cancer. Larger studies with longer follow-up are needed to further investigate this approach.
\end{abstract}

Keywords: McKeown; uniportal thoracoscopy; esophageal cancer

Submitted Mar 28, 2017. Accepted for publication Oct 10, 2019.

doi: $10.21037 /$ jtd.2019.11.07

View this article at: http://dx.doi.org/10.21037/jtd.2019.11.07

\section{Introduction}

Esophagectomy remains the core method in the multimodality treatment approach and offers a potential therapeutic schedule for patients with resectable esophageal carcinoma $(1,2)$. However, traditional open esophagectomy is associated with a significant high risk of operative morbidity and mortality (3-5). In 1992, minimally invasive esophagectomy (MIE) was first reported by Shimi and colleagues (6) as a thoracoscopic esophagectomy procedure, and since then, many centers have started modifying minimally invasive techniques to esophagectomy for malignancy (7-9). While some centers have preferred hybrid techniques utilizing thoracoscopy and laparotomy or laparoscopy and thoracotomy $(10,11)$, others have implemented a less invasive approach which 


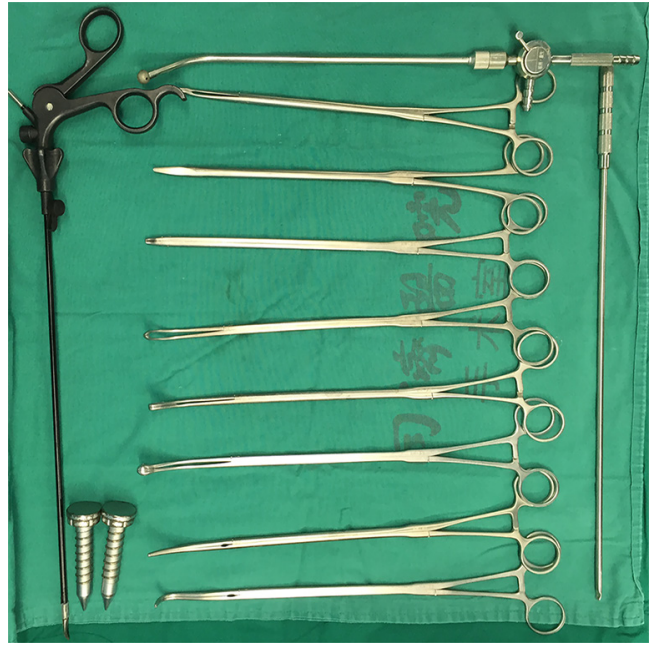

Figure 1 The instrument used in our surgery.

includes laparoscopic/thoracoscopic Ivor-Lewis resection, totally laparoscopic transhiatal esophagectomy, and MIE (McKeown) (12-14). Although has still been concern over the oncological outcomes of MIE, several meta-analyses have proclaimed that MIE has lower postoperative morbidity compared with traditional open procedures (15-17).

MIE typically entails video-assisted thoracoscopic surgery (VATS) with two to four incisions or ports. However, with the improvement of VATS techniques, recent studies have suggested that uniportal incision VATS is a promising treatment for performing MIE (18). This procedure involves less surgical incisions and is associated with reduced postoperative pain and complications compared with VATS. Similar results were observed in the treatment of non-small cell lung cancer (NSCLC), especially in uniportal VATS lobectomy $(19,20)$. In our institution, we take the MIE (McKeown) from May 2007, which was a combined laparotomy and thoracoscopic method with a neck anastomosis. Since Oct 2014, we have performed uniportal VATS lobectomy in the treatment of NSCLC. To minimize postoperative pain and perioperative complications, we modified the MIE McKeown procedure with uniportal thoracoscopy for upper or middle esophageal cancer. Here, we describe our initial experience and preliminary results.

\section{Methods}

\section{Patients}

This study was approved by the Ethics Committee of the Tongji Hospital, Tongji Medical College, Huazhong University of Science and Technology. Written informed consent was obtained from all patients before the surgical operation. Consecutive patients with esophageal carcinoma who underwent esophagectomy between March 2015 and May 2016 were evaluated and enrolled in study based on the following eligibility criteria: (I) histopathological confirmed esophageal carcinoma; (II) without obvious pulmonary/heat/renal failure or other basic disease; (III) esophageal tumors located in the upper or middle site; (IV) Clinical TNM range from T1N0M0 to T3N1M0; (V) no neoadjuvant therapy; (VI) no prior surgery involving the abdominal or thoracic cavity. Patients were excluded based on the following criteria: (I) with the severe underlying disease; (II) the tumor in serious condition (T4); (III) a wide range of pleural adhesions; (IV) received palliative resection. Based on these criteria, the study enrolled 44 patients for analysis.

The routine preoperative evaluation included chest radiographs, Doppler ultrasonography examinations of the abdomen, computed tomography scans of the chest and upper abdomen, barium swallow examinations, endoscopy with biopsy, electrocardiograms, pulmonary function tests, complete blood counts, and blood biochemistry screening containing liver and renal function evaluations. All patients underwent combined uniportal videoassisted thoracoscopic and laparoscopic esophagectomy and cervical anastomosis with curative intent. The TNM staging system of the American Joint Committee on Cancer (AJCC Staging Manual, 7th edition) was staged for patients postoperatively.

\section{Operating instruments}

The operating instruments included an Olympus highdefinition (HD) thoracoscopic surgical system (Olympus Medical Systems Corp., Tokyo, Japan); a 30 , 5/10-mm HD video camera; an endoscopic linear cutter; a flexible endoscopic stapler; and an Olympus ultrasound knife (Olympus, Tokyo, Japan). Certain double-joint endoscopic instruments (dissecting forceps, grasping forceps, and others, Figure 1) and an electric coagulation hook were custom-made. We also used a $12 \mathrm{Fr}$ catheter, Hem-o-lok® ligature clips (Weck Closure Systems, Research Triangle Park, NC, USA), a custom-made endo-pouch, a soft suction catheter, and other standard laparoscopic instruments. Instruments for conventional open thoracotomy were prepared at the same time. 


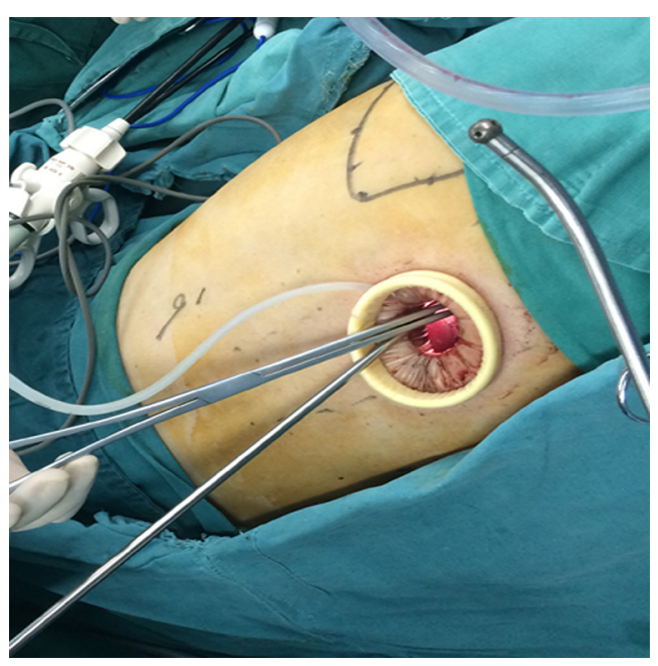

Figure 2 The location of the location.

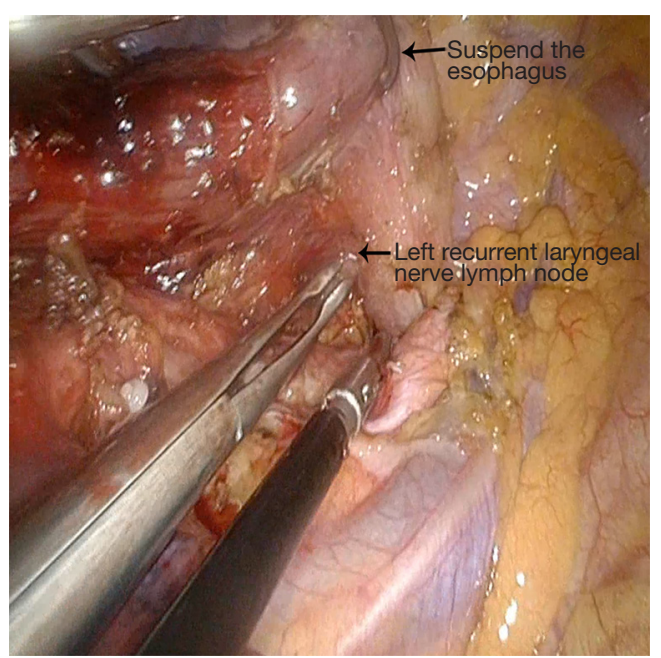

Figure 3 Dissection the left recurrent laryngeal nerve chain lymph node.

\section{Operative technique for modified McKeown procedure with uniportal thoracoscopy}

\section{Uniportal thoracoscopic phase}

Double-lumen endotracheal intubation anesthesia: The patients assumed a prone position. An incision, about $4 \mathrm{~cm}$ long, was made between the posterior and middle axillary line of the fifth intercostal space (Figure 2). A single-port thoracoscopy for thoracic esophageal and mediastinal lymph node dissection was used. Firstly, the right recurrent laryngeal nerve chain lymph node was dissociated, and the upper esophagus and broken arch of the azygos vein were

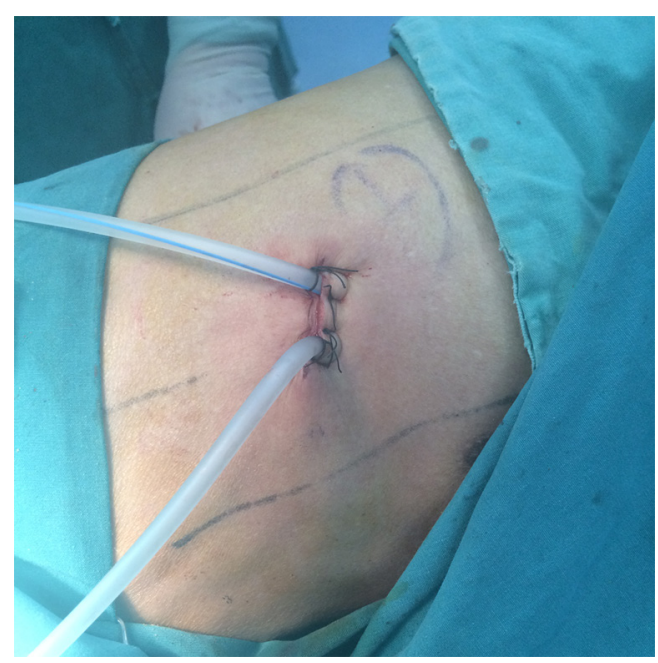

Figure 4 Modified two $16 \mathrm{~F}$ chest tube drainage.

freed. Secondly, the esophagus was dissociated from the azygos vein level to the diaphragmatic esophageal hiatus with a hook or ultrasound knife, and the thoracic duct was ligated, followed by the gradual dissection of the middlelower paraesophageal lymph nodes. Thirdly, the esophagus was cut off at the lower level with an Endo-GIA, the upper esophagus was suspended, revealing the tracheoesophageal groove, and the left recurrent laryngeal nerve chain lymph node was dissected (Figure 3). Finally, the two broken ends of the esophagus were connected with 1/0 VICRYL and placed in the esophageal bed. Two $16 \mathrm{~F}$ chest tube drainage is used in our center (Figure 4).

\section{Laparoscopic phase}

After the thoracoscopic phase, the patient was placed in a supine position. The abdominal ports were drilled, as shown in Figure 5. Firstly, the short gastric vessels were divided, and the right gastroepiploic vessels were preserved to mobilize the greater curvature of the stomach. Secondly, the abdominal tissue along the lesser curvature of the stomach was dissociated to the gastric cardia, and the left gastric artery and vein were divided. Thirdly, the dissociated tissue was removed through the incision, the gastric conduit was molded with the GIA linear stapler, and the gastric conduit was sutured in an uninterrupted fashion. Finally, the gastric conduit was placed back in situ with the connection from the thoracic VICRYL. The four remaining ports were closed, and the remaining single port A was temporarily closed. It is worth noting that lymph node dissection was performed during the laparoscopic operation. The vessels 


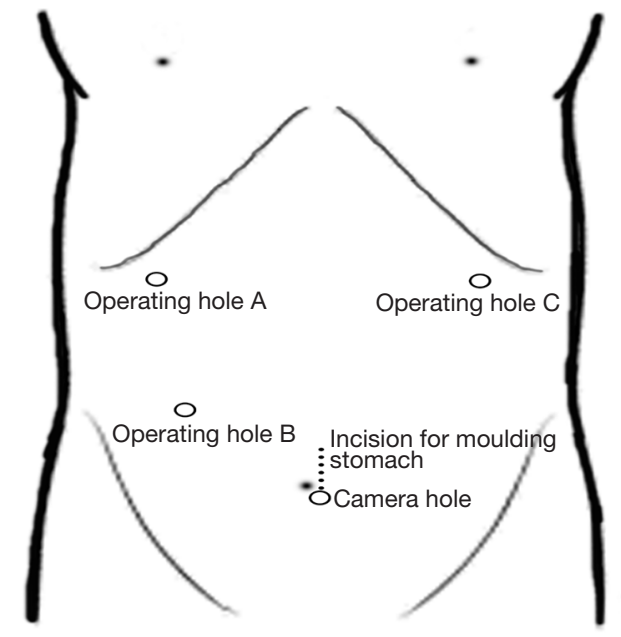

Figure 5 Location of abdominal ports.

were completely skeletonized, and the fatty tissues around the splenic artery, common hepatic artery, left gastric pedicle, and celiac axis were dissected.

\section{Cervical anastomosis}

An approximate 5 -cm oblique incision was made along the anterior border of the left sternocleidomastoid muscle. The cervical esophagus communicates with the right chest, which is needed to take note of the left recurrent laryngeal nerve. The neck esophagus was manually raised and transected, after which the distal end was connected to the pedestal of the circular stapler device. In this way, the gastric conduit was pulled up to the left neck through the posterior mediastinum, and assisted by the stapler to enable esophagogastric anastomosis. Notably, left neck lymphadenectomy was performed.

\section{Statistical analysis}

All statistical analyses were performed with dedicated analysis software (SPSS 17.0 statistical software package; SPSS, Chicago, IL, USA); descriptive statistics in this report are shown as mean $\pm \mathrm{SD}$.

\section{Results}

A total of 44 patients in this study underwent modified MIE with uniportal thoracoscopic for upper or middle esophageal cancer in our institution between March 2015 and May 2016 successfully. There were 24 males and 20 females with a mean age of 56.4 years and a range of $43-74$ years. There was no intraoperative complications or conversion to open surgery. The mean operating time was $408 \pm 34$ minutes, ranging from 394 to 495 minutes; the mean estimated blood loss was $245 \pm 102 \mathrm{~mL}$, ranging from 100 to $450 \mathrm{~mL}$; the meantime of thoracic operation was $163 \pm 16$ minutes, ranging from 135 to 199 minutes; the mean of dissected lymph nodes was $24 \pm 6$, ranging from 14 to 36; the mean number of lymph nodes dissected by uniportal video-assisted thoracoscopic was $12 \pm 2$, ranging from 9 to 15; the median of the hospital stay was 21 days, ranging from 14 to 40 days; no perioperative blood transfusion was required; all patients were extubated in the operating room. We did not have any intraoperative complications (e.g., massive hemorrhage, esophageal injury of the circular stapler device, pulmonary injury, and incomplete esophageal donut). Postoperative pathological diagnosis indicated that all patients had esophageal squamous cell carcinomas with R0 resection. Four patients had major postoperative complications. Two patients had a chest infection, and 2 patients had vocal cord palsy. The mean of follow-up was 2 months (range, 1-5 months), and no patients developed anastomotic stenosis or required endoscopic dilation.

\section{Discussion}

With the improvement and spread of VATS techniques, many centers have started modifying minimally invasive techniques to esophagectomy for malignancy $(7,8)$. At our institution, the MIE (McKeown) was initiated in May 2007 and has been perfected since Oct 2014. On the foundation of uniportal VATS lobectomy in the treatment of NSCLC, we modified the MIE McKeown procedure with uniportal thoracoscopy for upper or middle esophageal cancer to minimize postoperative pain and perioperative complications.

The important glittering in our study was the uniportal thoracoscopy and its accompanying products. From multiport to uniport, skills and protocols are changing and modifying. The incision is located at the posterior axillary line of the fifth intercostal space and is about $4 \mathrm{~cm}$ long. This incision provides a proper angle for thoracoscopic instruments due to the esophagus being located at the posterior mediastinum, which is different from the uniportal VATS lobectomy in the treatment of NSCLC (20). Meanwhile, the reduction of incisions increases the difficulty of endoscopic operation, and the protocol of 
thoracoscopic phase is rearranged. Many surgeons like to dissect lymph nodes first and then dissociate the esophagus. We have rearranged this protocol. After clear the right recurrent laryngeal nerve chain lymph node, we dissociate the esophagus and the accompanying lymph nodes. Then, we cut off the esophagus at the lower level with the EndoGIA before dissecting the left recurrent laryngeal nerve chain lymph node. With the suspension of the esophagus, the left recurrent laryngeal nerve chain is fully exposed and protected.

Furthermore, the reinforcement of gastric conduit and anastomosis reduces the occurrence of fistula. Ligation of the thoracic duct reduces the chylous fistula. Retention of the azygos vein is important for those patients who suffer from liver diseases, and can lower the complications. These protocols bring down the difficulties of operation and provide more dexterity in the views afforded.

In addition to the protocols, the instruments and operative skills were the important accompanying products which contributed to the shorter operation time, lower blood loss, and fewer postoperative complications. We use modified instruments and adjust their arrangement. A $30^{\circ}$, 10-mm HD thoracoscope provides a flexible view with high-quality frames and double-joint top-curved endoscopic instruments, creating a clear operating field and enhancing instrument use. All endoscopic instruments were custommade with the stem of the instruments being thin and long, which economizes the port and reduces instrument inference outside the thoracic cavity. In some cases, a soft suction catheter was used to aspirate "smoke" and blood rather than a laparoscopic aspirator. The arrangement of the instruments is also important. We preferred to rest the camera at the dorsal end of the incision directed towards the operating area and positioned instruments of low mobility at the distal end of the incision. The remaining larger incisional space allowed for easier operation and reduced interference. Finally, the crossing of hands and instruments is inevitable and beneficial, and grasping the instruments with the thumb and forefinger allows for the dominant hand to be in the ideal position most of the time, which is more comfortable and provides better instrument handling.

Our study has additional limitations and advantages. First, our study was preliminary, and the sample size was small, although our preliminary results showed similar performance in line with other previous studies $(3,10,16)$. In this report, a total of 44 patients successfully underwent modified McKeown MIE with uniportal thoracoscopic for upper or middle esophageal cancer without any intraoperative complications or conversion to thoracotomy or laparotomy. We had rigorous criteria, which is our top priority. Those who had severe tumors or underlying diseases were excluded from our study. Secondly, our attempts were based on our treatment of NSCLC and the skills of traditional MIE (McKeown). These experiences enable us to have a good command of the relevant surgical skills and anatomy which led to less blood loss and redundant damage of the tissue. However, this modified surgical protocol requires a skilled and experienced surgeon, postoperative manage is another benefit of our particular approach. The uniportal incisions simplify the management of operative wounds, and the chest tube drainage was revised from $16 \mathrm{Fr}$ tube to $30 \mathrm{Fr} / 34 \mathrm{Fr}$, which eases the pain of surgery with the same effects as $30 \mathrm{Fr} / 34 \mathrm{Fr}$ chest tube drainage (21). The uniportal incision and modified chest tube comfort the patients and provide a high degree of satisfaction.

Based on our results, MIE McKeown procedure with uniportal thoracoscopic surgery is, in our opinion, a feasible and safe option for the treatment of selected upper or middle esophageal cancer patients. These results support the decision to continue with this technique, although additional information from prospective studies is still needed.

\section{Acknowledgments}

None.

\section{Footnote}

Conflicts of Interest: The authors have no conflicts of interest to declare.

Ethical Statement: The authors are accountable for all aspects of the work in ensuring that questions related to the accuracy or integrity of any part of the work are appropriately investigated and resolved. This study was approved by the Ethics Committee of the Tongji Hospital, Tongji Medical College, Huazhong University of Science and Technology. Written informed consent was obtained from all patients before the surgical operation.

\section{References}

1. D'Journo XB, Thomas PA. Current management of esophageal cancer. J Thorac Dis 2014;6 Suppl 2:S253-64. 
2. Luketich JD, Pennathur A, Awais O, et al. Outcomes after minimally invasive esophagectomy: review of over 1000 patients. Ann Surg 2012;256:95-103.

3. Sihag S, Kosinski AS, Gaissert HA, et al. Minimally Invasive Versus Open Esophagectomy for Esophageal Cancer: A Comparison of Early Surgical Outcomes From The Society of Thoracic Surgeons National Database. Ann Thorac Surg 2016;101:1281-8; discussion 1288-9.

4. Smithers BM, Gotley DC, Martin I, et al. Comparison of the outcomes between open and minimally invasive esophagectomy. Ann Surg 2007;245:232-40.

5. Yerokun BA, Sun Z, Jeffrey Yang CF, et al. Minimally Invasive Versus Open Esophagectomy for Esophageal Cancer: A Population-Based Analysis. Ann Thorac Surg 2016;102:416-23.

6. Shimi SM, Nathanson LK, Cuschieri A. Thoracoscopic long oesophageal myotomy for nutcracker oesophagus: initial experience of a new surgical approach. Br J Surg 1992;79:533-6.

7. Senkowski CK, Adams MT, Beck AN, et al. Minimally invasive esophagectomy: early experience and outcomes. Am Surg 2006;72:677-83; discussion 683.

8. Nguyen NT, Slone J, Wooldridge J, et al. Minimally invasive esophagectomy without the use of postoperative nasogastric tube decompression. Am Surg 2009;75:929-31.

9. Pan H, Hu X, Yu Z, et al. Minimally invasive esophagectomy-left neck anastomosis. J Thorac Dis 2013;5:910-1.

10. Bjelovic M, Babic T, Spica B, et al. Could hybrid minimally invasive esophagectomy improve the treatment results of esophageal cancer? Eur J Surg Oncol 2016.

11. Scarpa M, Cavallin F, Saadeh LM, et al. Hybrid minimally invasive esophagectomy for cancer: impact on postoperative inflammatory and nutritional status. Dis Esophagus 2016;29:1064-70.

12. Sarkaria IS, Rizk NP, Finley DJ, et al. Combined thoracoscopic and laparoscopic robotic-assisted minimally

Cite this article as: Wang Q, Ping W, Cai Y, Fu S, Fu X, Zhang N. Modified McKeown procedure with uniportal thoracoscope for upper or middle esophageal cancer: initial experience and preliminary results. J Thorac Dis 2019;11(11):4501-4506. doi: 10.21037/jtd.2019.11.07 invasive esophagectomy using a four-arm platform: experience, technique and cautions during early procedure development. Eur J Cardiothorac Surg 2013;43:e107-15.

13. Ai B, Zhang Z, Liao Y. Laparoscopic and thoracoscopic esophagectomy with intrathoracic anastomosis for middle or lower esophageal carcinoma. J Thorac Dis 2014;6:1354-7.

14. Zhai C, Liu Y, Li W, et al. A comparison of short-term outcomes between Ivor-Lewis and McKeown minimally invasive esophagectomy. J Thorac Dis 2015;7:2352-8.

15. Biere SS, Maas KW, Bonavina L, et al. Traditional invasive vs. minimally invasive esophagectomy: a multi-center, randomized trial (TIME-trial). BMC Surg 2011;11:2.

16. Biere SS, Cuesta MA, van der Peet DL. Minimally invasive versus open esophagectomy for cancer: a systematic review and meta-analysis. Minerva Chir 2009;64:121-33.

17. Oor JE, Wiezer MJ, Hazebroek EJ. Hiatal Hernia After Open versus Minimally Invasive Esophagectomy: A Systematic Review and Meta-analysis. Ann Surg Oncol 2016;23:2690-8.

18. Guo $\mathrm{W}, \mathrm{Ma} \mathrm{L}$, Zhang $\mathrm{Y}$, et al. Totally minimally invasive Ivor-Lewis esophagectomy with single-utility incision video-assisted thoracoscopic surgery for treatment of midlower esophageal cancer. Dis Esophagus 2016;29:139-45.

19. Jutley RS, Khalil MW, Rocco G. Uniportal vs standard three-port VATS technique for spontaneous pneumothorax: comparison of post-operative pain and residual paraesthesia. Eur J Cardiothorac Surg 2005;28:43-6.

20. Reinersman JM, Passera E, Rocco G. Overview of uniportal video-assisted thoracic surgery (VATS): past and present. Ann Cardiothorac Surg 2016;5:112-7.

21. Kim SS, Khalpey Z, Daugherty SL, et al. Factors in the Selection and Management of Chest Tubes After Pulmonary Lobectomy: Results of a National Survey of Thoracic Surgeons. Ann Thorac Surg 2016;101:1082-8. 